\title{
EDITORIAL
}

\section{Randomised controlled trials in cystic fibrosis: what, when and how?}

\author{
P.D. Sly*, R.S. Ware*,\# , N. de Klerk ${ }^{\top}$ and S.M. Stick ${ }^{+}$
}

$\mathbf{T}$ he major morbidity and mortality from cystic fibrosis (CF) comes from progressive lung disease with bronchiectasis leading to respiratory failure. There is no doubt that survival of CF patients has increased progressively through the latter part of the 20th and the early part of the 21st centuries, and that the quality of life for patients with $\mathrm{CF}$ has increased substantially. However, CF still remains a serious life-shortening disease. Indeed, the most recent data from national $\mathrm{CF}$ registries show that the slope of survival curves [1] and the rate of loss of lung function have not changed at all, at least beyond childhood. Potential reasons for this are that CF lung disease begins in early life and that while current treatments may increase the health of children, they do little, if anything, to prevent the onset or progression of destructive lung disease, nor the inevitable onset of chronic respiratory failure.

Data from a number of sources have demonstrated that $\mathrm{CF}$ lung disease begins very early in life [2-6]. Arguably, the best data come from the Australian Respiratory Early Surveillance Team for Cystic Fibrosis (AREST CF), where essentially all children diagnosed with $\mathrm{CF}$ in a geographically defined region of Victoria and in Western Australia following detection by newborn screening are assessed soon after diagnosis and annually until 6-7 yrs of age [7]. These data show that a substantial proportion of infants have evidence of pulmonary inflammation and infection at 3 months of age, even in the absence of clinically apparent lung disease [8]. Structural lung disease, including bronchiectasis, is prevalent in infants and young children with CF [8-10]; the major risk factors being neutrophilic inflammation, the presence of free neutrophil elastase activity in the lungs and pulmonary infection, especially with Pseudomonas aeruginosa $[8,9]$. None of the current treatment strategies in routine use in paediatric CF clinics specifically target neutrophilic inflammation or the primary prevention of structural lung disease. Our data also suggest that once present, these structural abnormalities are progressive in the vast majority of children (AREST CF, unpublished observations).

\footnotetext{
*Queensland Children's Medical Research Institute, University of Queensland, "School of Population Health, University of Queensland, Brisbane, "Telethon Institute for Child Health Research, Centre for Child Health Research, University of Western Australia, and ${ }^{+}$Dept of Respiratory Medicine, Princess Margaret Hospital for Children, Perth, Australia.

CORRESPONDENCE: P.D. Sly, Queensland Children's Medical Research Institute, University of Queensland, Level 4, Foundation Building, Royal Children's Hospital, Herston, Brisbane, Australia E-mail: p.sly@uq.edu.au
}

A number of exciting new therapies for $\mathrm{CF}$ are currently in development and some have reached the clinical trial stage. These include: a variety of agents designed to correct or overcome genetic abnormalities in the production of the cystic fibrosis transmembrane conductance regulator or to improve its function as a chloride channel, and new strategies to overcome dehydration of the epithelial surface liquid (ESL) layer. Although these strategies may be more effective treatments for CF by improving electrolyte balance in the ESL, none are likely to reverse established bronchiectasis. The usual paradigm in drug development is to start clinical trials in adults with established disease and, if the initial studies are encouraging, move to children. However, this method has consigned drugs that may have been effective in preventing early disease in children to the pharmaceutical dustbin [11] when they failed to improve established disease in adults. Clearly a new approach is needed.

By their very nature, trials designed to test therapies to prevent disease in early life are going to be difficult to conduct. The major difficulties include: asking the appropriate research question; having the appropriate outcome variables; including a long enough period of follow-up to ensure that the disease has been prevented; and requiring a large sample size to have the power to demonstrate what may be relatively small effects. In addition, such trials will be expensive and will not suit the often short-term goals of the marketing departments of the pharmaceutical industry.

Although the difficulties in designing and carrying out trials of primary prevention strategies in CF may be substantial, they are not insurmountable. The data required to ask the appropriate research questions may already exist, e.g. in national CF data registries. In the current edition of the European Respiratory Journal, DE BОЕCK et al. [12] report data from the Belgian national CF data registry that show a small but significant decrease in lung function decline, expressed as forced expiratory volume in $1 \mathrm{~s}$ (FEV1) \% predicted, in children taking regular inhaled corticosteroids. This follows an earlier report with similar findings based on North American CF registry data [13]. The data from Belgium show that while there is a beneficial effect overall, the benefit from inhaled corticosteroids was restricted to children between the ages of 6 and 12 yrs; however, no data were available from children under the age of 6 yrs [12]. The registry data demonstrated that the mean decline in FEV1 \% pred in children aged 6-12 yrs was $1.37 \%$. The authors calculated that a study powered to show a $1 \%$ pred difference in the rate of lung function decline, 
assuming four measurements per year over 4 yrs, would require 242 children per study arm [12]. This report [12] shows the potential use of carefully collected registry data in posing appropriate research questions and aiding in study design. However, national registries contain few data that could be used to inform studies designed to prevent the onset of structural lung disease. In addition, the sample size required for the suggested study would require a major, expensive, international collaborative effort.

Well conducted surveillance studies, either cross-sectional or systematic longitudinal studies in representative populations, can also provide data on which to base a clinical trial. Crosssectional and longitudinal data from the AREST CF surveillance programme show that the prevalence of radiologically defined bronchiectasis at the age of 3 yrs is between 30 and $70 \%[8,9]$ (AREST CF, unpublished observations). Determining sample size based on hypothesis-testing procedures using conventional frequentist statistical methods, a study designed to decrease the prevalence of bronchiectasis at 3 yrs of age by $50 \%$ by treating from diagnosis would require 58 children per group $(\mathrm{P} 1=0.5$, $\mathrm{P} 2=0.25, \beta=0.8, \alpha=0.05)$. This type of study is not achievable within a single centre within a reasonable timeframe, but may be achievable with a national, multicentre approach. However, alternate approaches may facilitate such trials, either requiring fewer subjects or using international collaborations.

One such approach is to make use of data collected from longitudinal studies. The AREST CF surveillance programme includes computed tomography (CT) scans performed soon after diagnosis and annually until 6 yrs of age [9]. Using the methods of LEON [14], by including bronchiectasis data at 12 months and 3 yrs, and assuming an intraclass correlation coefficient (ICC) as high as $0.8,52$ children per group would give $80 \%$ power to detect a $50 \%$ difference between groups if the proportion of children with bronchiectasis in the placebo group is $0.5(\alpha=0.05)$. If the ICC is lower, the required group sizes decrease, being 41 for an ICC of 0.40 and 35 for an ICC of 0.20 . As most children who are going to acquire bronchiectasis by 5 yrs of age do so between 1 and 3 yrs, the ICC may be quite low between these ages.

It is also possible to move beyond binary outcomes and examine the extent of bronchiectasis in each child. Currently, the AREST CF scans are evaluated by examining each zone of the lung (right upper lung, right middle lung, right lower lung, left upper lung, Lingula and left lower lung) separately and assigning each a value between 0 and 2, where 0 represents no dilated airways, 1 is $\leqslant 50 \%$ of airways visible in a dilated zone and 2 is $\geqslant 50 \%$ of airway visible in a dilated zone, giving a range of $0-12$. Thus, we can calculate a bronchiectasis extent cumulative score (BECS) over two scans with a range from 0 to 24. We have examined preliminary data from 38 children with three CT scans until 3 yrs of age and found that the total BECS for the 38 children was 169 , with an average of 4.4. Assuming each child's score can be approximated with a Poisson distribution with a mean of 4.4, a trial with groups of 58 children will have $80 \%$ power to detect a difference between prevention therapies of a BECS of 1.0 (i.e. 3.4 versus 4.4), with $\alpha=0.05$ (AREST CF, unpublished observations).

Almost all therapies that have progressed to the clinical trial stage have some form of preliminary data available. In the current example, these data may come from national data registries or from surveillance programmes. Frequentist sample size calculations incorporate this prior information in the design phase of the trial. However, Bayesian statistics can take advantage of prior information at both the design and analysis phases of a study. Bayesian analysis uses Bayes' Theorem to formally combine prior information (represented as a probability distribution) with current information (i.e. the results of an ongoing randomised clinical trial) to produce a posterior distribution for each quantity of interest; in our case the prevalence of bronchiectasis at 3 yrs of age under different prevention therapies. The Bayesian approach allows for the updating of the researcher's state of knowledge as data accumulate. Consequently, adaptive trial designs can be used to potentially reduce the size of a trial, e.g. a trial can be ended at the point when enough information has been collected to answer the primary research question. While an adaptive approach requires extensive pre-planning, it often increases efficiency. At the study design phase, there is often uncertainly surrounding the sample size calculations, particularly in terms of an estimate of the variability of the primary outcome. If the outcome turns out to have more variation than expected, the study may turn out to be underpowered; if the outcome has less variation than expected, then researchers may collect data on more individuals than required. However, in a Bayesian analysis the variability is learned as the study progresses and an adaptive design allows for the study to be terminated or continued as appropriate. In longitudinal studies, measurements taken early in the study period may be used to predict final outcomes, potentially decreasing overall study length. For example, if there is sufficiently strong association between progression of bronchiectasis at 12 and 36 months, then data from participants enrolled earlier in the study (and who have both 12- and 36-month measures) can be used to predict progression of bronchiectasis for those enrolled later in the study who have data recorded at only 12 months.

Another strategy includes multiple sites conducting their own trial, using local funding, but ensuring that data can be combined with other sites by using "harmonised" protocols. The ultimate example of this is where every site uses exactly the same protocol under a "protocol franchising" arrangement, where a lead site develops the protocol and associated trial documentation (possibly in collaboration with investigators from other sites) and local sites adopt this protocol completely, obtaining local ethics permission and funding, and following all aspects of the protocol. Data from the trial sites would be transferred to a single data management and analysis site for compilation. However, the concept of harmonised protocols does allow some variability in procedures across sites. A unified core data set is required, but then sites with specific expertise could collect additional data, essentially as sub-studies to the main trial. In the case of a trial designed to demonstrate the efficacy of treatment $X$ at preventing bronchiectasis, all sites may be required to undertake CT scans at 3, 12 and 36 months of age to provide the primary outcome data. Sites with expertise in bronchoalveolar lavage (BAL) could provide mechanistic data for how treatment $X$ prevents bronchiectasis and sites with expertise in infant lung function testing could provide data on a subset showing whether the successful prevention of bronchiectasis was reflected in 
improvements in lung function. Essentially, the only requirement on trial sites is that they follow the core and supplementary protocols (BAL, lung function testing, etc.) and that they interact with the lead site to obtain blinded randomisation codes, which must be stratified by site, and are prepared to send blinded data to the coordination site. While this approach sounds simple, it does require an adequately resourced coordination site and good will from all parties [15].

We are on the verge of a potentially exciting era in CF research and management, but one that will require a change in approach from that which has governed the development, testing and introduction of novel therapies designed to prevent the destructive lung disease characteristic of CF. Clinicians and academics in the CF field will need to take the lead, with industry as a willing partner, for the new horizon to be reached.

\section{STATEMENT OF INTEREST}

None declared.

\section{REFERENCES}

1 Cystic Fibrosis Foundation. Patient Registry: Annual Data Report 2008. Bethesda, Cystic Fibrosis Foundation, 2008.

2 Armstrong DS, Grimwood K, Carlin JB, et al. Bronchoalveolar lavage or oropharyngeal cultures to identify lower respiratory pathogens in infants with cystic fibrosis. Pediatr Pulmonol 1996; 21 267-275

3 Armstrong DS, Grimwood KK, Carlin JB, et al. Lower airway inflammation in infants and young children with cystic fibrosis. Am J Respir Crit Care Med 1997; 156: 1197-1204.
4 Armstrong DS, Grimwood KK, Carzino RR, et al. Lower respiratory infection and inflammation in infants with newly diagnosed cystic fibrosis. BMJ 1995; 310: 1571-1572.

5 Khan TZ, Wagener JS, Bost TT, et al. Early pulmonary inflammation in infants with cystic fibrosis. Am J Respir Crit Care Med 1995; 151: $1075-1082$.

6 Nixon GM, Armstrong DS, Carzino R, et al. Early airway infection, inflammation, and lung function in cystic fibrosis. Arch Dis Child 2002; 87: 306-311.

7 Australian Respiratory Early Surveillance Team for Cystic Fibrosis. www.arestcf.org Date last accessed: January 14, 2011. Date last updated: August 8, 2010.

8 Sly PD, Brennan S, Gangell C, et al. Lung disease at diagnosis in infants with cystic fibrosis detected by newborn screening. Am J Respir Crit Care Med 2009; 180: 146-152.

9 Stick SM, Brennan S, Murray C, et al. Bronchiectasis in infants and preschool children diagnosed with cystic fibrosis after newborn screening. J Pediatr 2009; 155: 623-628.

10 Mott LS, Gangell CL, Murray CP, et al. Bronchiectasis in an asymptomatic infant with cystic fibrosis diagnosed following newborn screening. J Cyst Fibros 2009; 8: 285-287.

11 Holt PG, Sly PD, Martinez FD, et al. Drug development strategies for asthma: in search of a new paradigm. Nat Immunol 2004; 5: 695-698.

12 De Boeck K, Vermeulen F, Wanyama S, et al. Inhaled corticosteroids and lower lung function decline in young children with cystic fibrosis. Eur Respir J 2011; 37: 1091-1095.

13 Ren C, Pasta D, Rasouliyan L, et al. Relationship between inhaled corticosteroid therapy and rat of lung function decline in chidlren with cystic fibrosis. J Pediatr 2008; 153: 746-751.

14 Leon A. Sample-size requirements for comparisons of two groups on repeated observations of a binary outcome. Eval Health Prof 2004; 27: 34-44.

15 Golding J. The case for a coordinating centre for birth cohort studies. Paediatr Perinat Epidemiol 2009; 23: 226-229. 